\title{
Registros de Mantodea (Insecta) coletados à luz no dossel da floresta, na torre do km 14 do núcleo ZF-2, Manaus, Brasil'
}

\author{
Yana Karlla Lessa Alves DANTAS², José Albertino RAFAEL ${ }^{3,4}$, Catarina da Silva MOTTA ${ }^{3}$, Antonio Arnovis \\ AGUDELO $^{5}$, Francisco Felipe XAVIER FILHO ${ }^{3}$
}

\begin{abstract}
RESUMO
Foram realizadas coletas mensais de insetos de janeiro a dezembro de 2004, durante três noites de transição lunar minguante/ nova, das 18:00 às 06:00 horas. Os espécimes foram capturados em um lençol iluminado com lâmpada de 250 watts, luz mista de vapor de mercúrio e lâmpada de 20 watts black light (BL) e black light bulb (BLB). A armadilha foi montada a 45 metros de altura numa torre metálica de 50 metros, que ultrapassa a maioria das copas das árvores, num platô de terra firme, na bacia do rio Cuieiras, Manaus, Amazonas, Brasil. Foram coletados 23 espécies de Mantodea, sendo Chaeteessidae (1 espécie); Mantoididae (2); Mantidae (15); Thespidae (2) e Acanthopidae (3). Seis espécies são novas e serão descritas oportunamente nos seguintes gêneros: Cardioptera Burmeister, 1838, Phyllovates Kirby, 1904, Pseudovates Saussure, 1869, Stagmomantis Saussure, 1869, Stagmatoptera Burmeister, 1838 e Metilia Stal, 1877. Três espécies registradas para o Brasil sem uma região determinada estão sendo registradas para a Amazônia brasileira: Heterovates pardalina Saussure, 1872, Macromantis ovalifolia (Stoll, 1813) e Photina reticulata (Burmeister, 1838). Quatro registros são novos para o estado do Amazonas: Angela guianensis Rehn, 1906, Photina gracillis Giglio-Tos, 1915, Raptrix perspicua (Fabricius, 1787) e Vates festae Gigio-Tos, 1914. Os números de indivíduos, em cada coleta mensal, são apresentados para cada espécie.
\end{abstract}

PALAVRAS-CHAVE: Armadilha luminosa; coleta noturna; dossel; floresta amazônica; Mantodea.

\section{Records of Mantodea (Insecta) collected with light trap at 45 meters height over an Amazon forest canopy, at ZF-2 nucleus, Manaus, Brazil}

\section{ABSTRACT}

Insect collections were carried out monthly from January to December of 2004, during three nights of lunar transition third quarter moon/new moon from 18 p.m. to 6 a.m. They were collected over a vertical white sheet illuminated by a 250 watts mixed light and a 20 watts black light (BL) and black light bulb (BLB) lamps. The light trap was mounted at 45 meters height in a metallic tower of 50 meters height, in a "platô de terra firme", in the Cueiras River basin, in Manaus, Amazonas, Brazil. It was collected 23 species of Mantodea: Chaeteessidae (1 species); Mantoididae (2); Mantidae (15); Thespidae (2) and Acanthopidae (3). Six new species were registered and they will be described opportunely in the following genera: Cardioptera Burmeister, 1838; Phyllovates Kirby, 1904; Pseudovates Saussure, 1869; Stagmomantis Saussure, 1869; Stagmatoptera Burmeister, 1838 and Metilia Stal, 1877. Three species previously recorded to an indetermined Brazilian region are being recorded to Brazilian Amazon: Heterovates pardalina Saussure, 1872; Macromantis ovalifolia (Stoll, 1813) and Photina reticulata (Burmeister, 1838). Four records are new for the Amazonas state: Angela guianensis Rehn, 1906; Photina gracillis Giglio-Tos, 1915; Raptrix perspicua (Fabricius, 1787) and Vates festae Gigio-Tos, 1914. The specimens number collected monthly are presented for each species.

KEYWORDS: Light trap, Nocturnal collection, Canopy, Amazonian forest, Mantodea.

\footnotetext{
1 Projeto "Biodiversidade de Arthropoda no manejo e conservação: um modelo amazônico", processo 472978/2003-9 financiado pelo Conselho Nacional de Desenvolvimento Científico e Tecnológico (CNPq).

2 Bolsista de Iniciação Científica do CNPq. 501402/2004-7. e-mail: ykla@ig.com.br

3 Instituto Nacional de Pesquisas da Amazônia, Manaus, Amazonas, C.P. 478, 69011-970, Manaus, Amazonas, Brasil. email: jarafael@inpa.gov.br; motta@inpa.gov.br; ffelipe@inpa.gov.br

${ }^{4}$ Bolsista Produtividade do CNPq.

${ }^{5}$ Instituto de Investigación de Recursos Biológicos Alexander von Humboldt, Alexander von Humboldt, DG. 27, nº 15-09, PBX: 316-7880, Bogotá / Colômbia e-mail: mantidarvm@yahoo.com
} 


\section{INTRODUÇÃO}

A ordem Mantodea constitui um grupo pequeno e cosmopolita, com cerca de 2.300 espécies descritas em todo o mundo (Ehrmann, 2002) e 425 para a região Neotropical (Agudelo et al., 2007). No Brasil foram registradas 259 espécies (Jantsch, 1999) e dessas 27 são citadas para o estado do Amazonas (Jantsch \& Corseuil, 1988).

Os mantódeos, ou louva-a-deus, são pouco conhecidos na região Neotropical. Isso se deve, possivelmente, ao baixo esforço de coleta, à baixa densidade populacional, ao mimetismo de suas espécies e à escassa bibliografia produzida pelos poucos especialistas do grupo (Cerdá, 1993; VelásquezTibatá, 2000; Escobar, 2002). No estado do Amazonas nenhum estudo faunístico foi realizado com esse grupo. Os existentes são essencialmente taxonômicos, ainda restritos às descrições de espécies.

O dossel nas florestas tropicais possui uma estrutura complexa, com grande variedade de nichos (Santos et al., 2003) onde, pode-se destacar não somente a biomassa foliar das copas das árvores, como também a presença de outras formaçōes vegetais como as lianas e epífitas. Face à sua complexidade esse trabalho se torna importante por ser pioneiro, pois, se trata da primeira contribuição ao conhecimento da composição taxonômica, distribuição anual e abundância das espécies de Mantodea no estrato superior de uma floresta na região Amazônica, no município de Manaus.

\section{MATERIAL E MÉTODOS}

Os Mantódeos foram coletados de 18:00 às 06:00 horas, em uma área de floresta primária, com armadilha luminosa que consistiu de um lençol branco iluminado com lâmpada de 250 watts, luz mista de vapor de mercúrio e lâmpadas black light (BL) e black light bulb (BLB). A armadilha foi montada em nível superior ao da maioria das copas das árvores, a 45 metros de altura, em uma torre metálica com as dimensōes de $6 \times 6$ metros de largura $\times 50$ metros de altura, localizada num platô de terra firme ( $\left.2^{\circ} 35^{\prime} 21^{\prime \prime} \mathrm{S}, 60^{\circ} 06^{\prime} 55^{\prime \prime} \mathrm{W}\right)$ na bacia do Rio Cuieras, Estação Experimental de Silvicultura Tropical do Instituto Nacional de Pesquisas da Amazônia (INPA), $\mathrm{km} 14$ do núcleo ZF-2, Amazonas. O estudo da composição florística da área foi realizado por Carneiro (2004) a cerca de cinco quilômetros da torre e é muito semelhante à existente nas proximidades da torre (Martins et al., 2006).

As coletas foram realizadas mensalmente, durante três noites, no período de transição lunar minguante/nova, de janeiro a dezembro de 2004, nas noites mais escuras. Os insetos foram coletados manualmente no lençol e sacrificados em frascos com acetato de etila. Foram montados com alfinetes entomológicos no local de coleta e trazidos ao laboratório para secagem em estufa e posterior etiquetagem. A identificação foi feita com auxílio dos trabalhos de Giglio-Tos (1927) e Terra (1995).

O material estudado está depositado na Coleção de Invertebrados do Instituto Nacional de Pesquisas da Amazônia (INPA).

\section{RESULTADOS E DISCUSSÃO}

Foram coletados 254 espécimes de Mantodea distribuídos em 5 famílias, 17 gêneros e 23 espécies. A lista de espécies, a densidade em cada coleta mensal e o número total de indivíduos constam na Tabela 1.

As espécies mais abundantes foram Parastagmatoptera serricornis Kirby, 1904 com 74 exemplares (29\%), coletada durante todo o ano, com maior densidade em janeiro e fevereiro (Tab. 1); Stagmatoptera sp. N. com 42 exemplares (16\%) e maior densidade em setembro; Vates festae Giglio-Tos, 1914 com 28 exemplares (11\%), Angela guianensis Rehn, 1906 com 19 exemplares (7\%), Raptrix perspicua (Fabricius, 1787) com 12 exemplares (5\%) e Vates pectinata Saussure, 1871 com 10 exemplares (4\%). As últimas quatro espécies foram coletadas em todos os meses, sem uma estação definida.

A coleta de fêmeas foi registrada para as espécies Heterovates pardalina Saussure, 1872 com dois exemplares (outubro e dezembro), Parastagmatoptera serricornis com dois exemplares (fevereiro e agosto), Vates festae e V. pectinata com um exemplar cada em setembro. A percentagem de fêmeas capturadas foi de 1,6\%, sugerindo que fêmeas de algumas espécies de Mantodea podem ser capturadas ocasionalmente nas armadilhas luminosas.

A coleta de 23 espécies de mantódeos no dossel da floresta pode ser um indício de que várias espécies habitam a copa das árvores e voam ao nível e sobre as copas das árvores para dispersão, possivelmente em busca de presas para sua alimentação e procura do sexo oposto para acasalamento. Muitas dessas espécies já foram coletadas nos estratos inferiores da floresta, demonstrando que tais espécies colonizam diferentes estratos vegetais, conforme dados nas etiquetas de espécimes depositados em coleçōes.

A influência atrativa da armadilha luminosa sobre espécimes de mantódeos dos estratos inferiores parece ser mínima. Foi observado ao nível do solo que o raio de ação da lâmpada montada a $45 \mathrm{~m}$ de altura, no ambiente de floresta, não atingia 50 metros de distância da base da torre, e, portanto, provavelmente pouco influenciou na atratividade de espécimes dos estratos inferiores na coleta realizada no dossel.

Seis espécies coletadas no dossel são novas e serão descritas oportunamente (Tabela 1).

Os seguintes registros são novos para o estado do Amazonas e Amazônia brasileira: os gêneros Cardioptera e Phyllovates, de ocorrência nas regiōes nordeste, sudeste, centro-oeste e 
Tabela 1 - Densidade das espécies de Mantodea coletadas no nível do dossel, com armadilha luminosa, durante três noites de transição lunar minguante/nova em todos os meses do ano 2004, na torre do km 14 da ZF-02, Manaus, Amazonas.

\begin{tabular}{|c|c|c|c|c|c|c|c|c|c|c|c|c|c|c|}
\hline \multirow{2}{*}{ Famílias } & \multirow{2}{*}{ Espécies } & \multicolumn{12}{|c|}{$\mathrm{N}^{0}$ de indivíduos / Mês } & \multirow{2}{*}{ Total } \\
\hline & & $\mathbf{J}$ & $\mathbf{F}$ & M & A & M & $\mathbf{J}$ & $\mathbf{J}$ & A & $S$ & 0 & $\mathbf{N}$ & D & \\
\hline CHAETEESSIDAE & Chaeteessa caudata Saussure, 1871 & & & & & 1 & & & & & & & & 1 \\
\hline \multirow[t]{2}{*}{ MANTOIDIDAE } & Mantoida brunneriana (Saussure, 1871) & & & & & & & 1 & & 1 & & & & 2 \\
\hline & M. luteola Westwood, 1889 & & 1 & 1 & 1 & & & 1 & 1 & & & & 1 & 6 \\
\hline \multirow[t]{15}{*}{ MANTIDAE } & Cardioptera sp. n. & & 1 & & 1 & 1 & & 2 & & 3 & & & 1 & 9 \\
\hline & Choeradodis rhomboidea (Stoll, 1813) & & & & & & & & & & & & 1 & 1 \\
\hline & Heterovates pardalina Saussure, 1872 & & 1 & & & & 1 & & 1 & & 1 & & 1 & 5 \\
\hline & Macromantis hyalina (De Geer, 1773) & & & & & & & 2 & & & & 2 & & 4 \\
\hline & M. ovalifolia (Stoll, 1813) & & & & & & & 1 & & & & & & 1 \\
\hline & Microphotina vitripennis Saussure, 1872 & & & & & 1 & & 1 & 3 & & & & 2 & 7 \\
\hline & Parastagmatoptera serricornis Kirby, 1904 & 10 & 19 & 3 & 3 & 1 & 4 & 8 & 10 & 3 & 2 & 2 & 9 & 74 \\
\hline & Photina gracillis Giglio-Tos, 1915 & & 1 & & & & & & 1 & & & & & 2 \\
\hline & P. reticulata (Burmeister, 1838) & & & & & & & & 2 & & & & & 2 \\
\hline & Phyllovates sp. n & & & & & & & 1 & 1 & & & & & 2 \\
\hline & Pseudovates sp. $\mathrm{n}$. & & & 1 & 1 & 1 & & 1 & 1 & & 2 & & & 7 \\
\hline & Stagmatoptera sp. $n$. & 3 & 2 & 4 & 4 & 2 & 3 & 5 & 4 & 8 & & 4 & 3 & 42 \\
\hline & Stagmomantis sp. $n$. & 2 & 1 & 1 & & & 1 & & 1 & 1 & & & 2 & 9 \\
\hline & Vates festae Giglio-Tos, 1914 & 8 & 2 & & 1 & & & 3 & 5 & 5 & & 2 & 2 & 28 \\
\hline & V. pectinata Saussure, 1871 & 1 & 1 & & & 2 & & 2 & 1 & 1 & & & 2 & 10 \\
\hline \multirow[t]{2}{*}{ THESPIDAE } & Angela guianensis Rehn, 1906 & 2 & 2 & 1 & 3 & 1 & 1 & & 6 & & 1 & 1 & 1 & 19 \\
\hline & A. peruviana Giglio-Tos, 1916 & & 2 & & & & 1 & & 1 & 1 & 1 & & & 6 \\
\hline \multirow[t]{3}{*}{ ACANTHOPIDAE } & Metilia brunneri (Saussure, 1871) & & 2 & & 1 & 1 & & & & & & & & 4 \\
\hline & Metilia sp. n. & & & & & & & & & & 1 & & & 1 \\
\hline & Raptrix perspicua (Fabricius, 1787) & 6 & 1 & & & & & & 4 & 1 & & & & 12 \\
\hline Total & & 32 & 36 & 11 & 15 & 11 & 11 & 28 & 42 & 24 & 8 & 11 & 25 & 254 \\
\hline
\end{tabular}

sul do Brasil (Terra, 1995; Jantsch, 1999; Ehrmann, 2002; Agudelo, 2004); as espécies Heterovates pardalina Saussure, 1872; Macromantis ovalifolia (Stoll, 1813) e Photina reticulata (Burmeister, 1838), as três anteriormente sem dados de procedência de localidades no Brasil.

Os seguintes registros são novos para o estado do Amazonas: as espécies Angela guianensis Rehn, 1906, antes assinalada nos estados do Maranhão e Pará (Jantsch, 1999; Ehrmann, 2002; Agudelo, 2004); Photina gracillis GiglioTos, 1815, antes assinalada nos estados de Roraima, Pará, Rio de Janeiro e Santa Catarina (Jantsch, 1999); Raptrix perspicua (Fabricius, 1787) antes nos estados do Amapá, Pará, Pernambuco, Espírito Santo, São Paulo, Santa Catarina e Rio Grande do Sul (Jantsch 1999; Agudelo 2004; Lombardo \& Marletta, 2004) e Vates festae Gigio-Tos, 1914, antes nos estados do Pará e Bahia (Jantsch 1999; Ehrmann 2002; Agudelo 2004).

Com esse trabalho o número de espécies para o estado do Amazonas foi elevado de 27 (Jantsch e Corseuil, 1988) para 36 espécies, mostrando que o estrato superior da floresta deve ser mais bem estudado para ampliar o conhecimento sobre os mantódeos na região amazônica.

\section{AGRADECIMENTOS}

Os autores agradecem aos colegas Simone Trovisco, Joseneide Câmara, Fábio Godoi, Fabrício Baccaro, Alexandre Ururahy Rodrigues, Marcelo Cutrim, Alexandre da Silva Filho, José Moacir Ribeiro pelo apoio recebido durante as coletas de campo.

\section{BIBLIOGRAFIA CITADA}

Agudelo, A.A. 2004. Mántidos de Colombia (Dictyoptera: Mantodea). In: Fernádez-C., Andrade-C., Amat-G. (Eds). Insectos de Colombia. Volumen III. Universidad Nacional de Colombia. Bogotá D.C. 604pp.

Agudelo, A.A.; Lombardo, F.; Jantsch, J.L. 2007. Checklist of the neotropical Mantids (Insecta, Dictyoptera, Mantodea). Biota Colombiana, 8(2): 105-158

Carneiro, V.M. C. 2004. Composição florística e análise estrutural de floresta primária de terra firme na bacia do rio Cuieiras, Manaus$A M$. Dissertação de Mestrado. Instituto Nacional de Pesquisas da Amazônia/Universidade do Amazonas, Manaus, Amazonas. $\mathrm{x}+67 \mathrm{pp}$.

Cerdá, F.J. 1993. Mantodea de Venezuela. Géneros y lista preliminar de especies. Parte I Mantoididae e Hymenopodidae. Revista de la Facultad de Agronomia, 19: 129-151. 
Ehrmann, R. 2002. Mantodea. Gottesanbeterinnen der Welt. Natur und Tier - Verlag GmbH. 519 pp.

Escobar, J.A.S. 2002. Lista de los Mantódea (Insecta) conocidos para Colômbia. Biota Colombiana, 3(1): 119-130.

Giglio-Tos, E. 1927. Das Tierreich. Orthoptera-Mantidae. Walter de Gruyter \& Co., Berlin. 707pp.

Jantsch, L.J.; Corseuil, E. 1988. Espécies de louva-a-deus (Insecta, Mantodea) do Rio Grande do Sul, Brasil. Revista Brasileira de Zoologia, 5(2): 221-243.

Jantsch, L. 1999. Estudos filogenéticos em mantódeos americanos (Insecta; Pterygota; Mantodea). Tese de Doutorado. Pontifícia Universidade Católica do Rio Grande do Sul, 137pp.

Lombardo, F; Marletta, A. 2004. Remarks on the genus Raptrix Terra, 1995 (Mantodea, Acontiothespinae) with a description of two new species. Journal of Orthoptera Research, 13(1): 21-33.

Martins U. R.; Galileo, M.H.M.; Santos-Silva, A.; Rafael, J.A. 2006. Cerambycidae (Coleoptera) coletados à luz a 45 metros de altura, no dossel da floresta amazônica, e a descrição de quatro espécies novas. Acta Amazonica, 36(2): 265-272.
Roy, R. 2002. Révision du genre néotropical Macromantis Saussure, 1871 (Dictyoptera, Mantidae). Bulletin de la Société Entomologique de France, 107(4):403-418.

Santos, G.B.; Marques, M.I.; Adis, J.; Musis, C.R. 2003. Artrópodos associados à copa de Attalea phalerata Mart. (Arecaceae), na região do Pantanal de Poconé, Mato Grosso, Brasil. Revista Brasileira de Entomologia, 47(2): 211-224.

Terra, P. 1995.- Revisão sistemática dos gêneros de louva-a-deus da região Neotropical (Mantodea). Revista Brasileira de Entomologia, 39(1): 13-94.

Velásquez-Tibatá, J. 2000. Heterovates pardalina Saussure, 1872. Nueva adición a la mantidofauna colombiana (Insecta: Mantodea) Boletín Científico Museo de Historia Natural Universidad de Caldas, 4: 98-101.

Recebido em 06/10/2006

Aceito em 29/12/2007 